\title{
Comparison of Laparoscopy and Laparotomy in Surgical Treatment of Ectopic Pregnancies: A 6-Year Experience at a Tertiary Center
}

\section{PInar YALÇIN BAHAT,a \\ (D) Gökçe TURAN, \\ (1) Berna ASLAN ÇETIN,a ibrahim POLAT ${ }^{a}$}

${ }^{a}$ Clinic of Gynecology and Obstetrics, Kanuni Sultan Süleyman Training and Research Hospital,

İstanbul

${ }^{b}$ Clinic of Gynecology and Obstetrics, Kırıkan State Hospital,

Hatay

Received: 24.05.2018

Received in revised form: 02.07 .2018

Accepted: 10.07 .2018

Available online: 31.08 .2018

\section{Correspondence:}

Gökçe TURAN

Kırıkhan State Hospital,

Clinic of Gynecology and Obstetrics,

Hatay, TURKEY

drgokceturan@gmail.com

\begin{abstract}
Objective: To investigate laparotomy and laparoscopic approaches in patients diagnosed with ectopic pregnancy (EP) and who underwent surgical treatment in our clinic during the last six years. Material and Methods: This study included 202 patients who underwent laparoscopic surgery and laparotomy for EP between January 2012 and January 2018 in our clinic. A comparison was made among the patients for age, gravidity, parity, mean $\beta$ human chorionic gonadotropin (HCG) levels on admission, risk factors for EP, and clinical findings. The contributions of these two treatments to the postoperative process were assessed. Results: The surgical intervention was performed on 202 patients who had an EP. Of these patients, 110 (54\%) underwent laparotomy and 92 (46\%) underwent laparoscopic surgery. No differences were found at a statistically significant level between the groups in age, gravidity, parity, curettage, gestational age at admission, mean $\beta$-HCG levels on admission, and preoperative and postoperative hemoglobin levels. Although the length of postoperative hospital stay was shorter at a statistically significant level in the laparoscopy group than in the laparotomy group, operation time was shorter at a statistically significant level in the laparotomy group than in the laparoscopy group. Conclusion: When surgical treatment is decided in patients with EP, laparoscopic surgery can be preferred more by considering the fact that it leads to less contamination, reduces hospital length of stay, and has a cost advantage over laparotomy. However, treatment methods can vary according to the facilities of clinics, availability of surgical instruments, and experience of physicians.
\end{abstract}

Keywords: Ectopic pregnancy; laparoscopy; laparotomy; risk factors

$\mathrm{E}$ ctopic pregnancy (EP) is the implantation and development of a fertilized ovum in any other area outside of the uterine cavity. The incidence of EP ranges from 1/1000 to $1 / 150$ pregnancies. ${ }^{1}$ EPs constitute $1-2 \%$ of all pregnancies and are among the most important morbidity and mortality reason in women of reproductive age. ${ }^{2}$ In recent years, EP can be diagnosed earlier by the use of transvaginal ultrasonography and the use of quantitative $\beta$-human chorionic gonadotropin (HCG) levels in routine in almost every clinic. This diagnosis leads to a decrease in the EP-related mortality rate. The diagnosis of patients without rupture allows them to benefit from medical or surgical treatment. ${ }^{3}$ Laparoscopic approaches are primarily preferred in the surgical treatment of EP in women who are hemodynamically stable. ${ }^{4}$ Laparoscopic treatment of EP has less intraoperative blood loss, less operational time, less analgesic necessity, less hospital stay, less recovery time, and economic cost advantages. ${ }^{5}$

The purpose of this study was to examine the laparotomy and laparoscopic modalities in patients with EP who received surgical treatment in 
our clinic during the last six years and to assess the contributions of these two surgical treatments to the postoperative process.

\section{MATERIAL AND METHODS}

We examined retrospectively 471 patients who were diagnosed with EP between January 2012 and January 2018 in the Obstetrics and Gynecology Clinic, University of Health Sciences, Kanuni Sultan Suleyman Training and Research Hospital, Istanbul, Turkey. From the patients enrolled in the study, the data of only patients undergoing surgical treatment were analyzed. Of the 471 patients with EP, 202 (42\%) underwent surgical treatment.

Surgical treatment was performed in patients who were hemodynamically unstable, had a ruptured EP during follow-up or on first admission after medical treatment, had any contraindication to methotrexate therapy, had concomitant intrauterine pregnancy, did not accept medical treatment, requested permanent contraception, and first received medical treatment but did not respond to medical treatment during follow-up. The patients who received surgery were separated into two groups: laparoscopic surgery and laparotomy. A comparison was made between these patients for age, gravidity, parity, mean $\beta$-HCG levels on admission, risk factors for EP, and clinical findings. All patients underwent a dilatation and curettage procedure. The EP diagnosis was verified with a pathological examination.

Statistical analyses were made by employing the Statistical Package for Social Sciences 20.0 (SPSS Inc.; Chicago, IL, USA). The KolmogorovSmirnov test was employed to investigate the data for normal distribution. In addition to descriptive statistical methods (mean, standard deviation) in the evaluation of normally distributed data, the independent sample t-test was used for binary comparisons. A p-value of $<0.05$ was considered statistically significant.

\section{RESULTS}

Of the 471 patients with EP, 202 (42\%) underwent surgical treatment. Of these patients, $110(54 \%)$ underwent laparotomy and 92 (46\%) underwent laparoscopic surgery. The surgical data of the two groups are listed in Table 1.

The mean age of patients was $32 \pm 5.1$ years in the laparotomy group and $33.1 \pm 4.9$ years in the laparoscopy group. No statistically significant differences were observed between the two groups for age, gravidity, parity, curettage, gestational age at admission, mean $\beta$-HCG levels on admission, preoperative and postoperative hemoglobin $(\mathrm{Hb})$ levels. Although the postoperative hospital stay was shorter at a statistically significant level in the laparoscopy group than in the laparotomy group, operation time was significantly shorter in the laparotomy group than in the laparoscopy group. Demographic data, operation time, length of hospital stay, preoperative and postoperative Hb levels of patients in the two groups are provided in Table 2.

When the reasons for the initial admission in the two groups were examined, the most common complaint found in both groups was abdominal pain. When the risk factors for EP in the two groups were examined, the most common risk factor found in both groups was previous abdominal surgery. However, no significant differences were detected between the groups for complaints at ad-

\begin{tabular}{|c|c|c|c|c|}
\hline \multirow[b]{2}{*}{ Salpingostomy } & \multicolumn{2}{|c|}{ Laparoscopy (n:92) (\%) } & \multicolumn{2}{|c|}{ Laparotomy (n:110) (\%) } \\
\hline & 10 & 10.8 & 8 & 7.2 \\
\hline Salpingectomy (including those ruptured during methotrexate therapy) & 78 & 84.7 & 96 & 87.2 \\
\hline Over wedge resection & 1 & 1.08 & 3 & 2.7 \\
\hline Cornual wedge resection & 2 & 2.1 & 1 & 0.9 \\
\hline Milking & 1 & 1.08 & 0 & 0 \\
\hline Scar resection & 0 & 0 & 2 & 1.8 \\
\hline
\end{tabular}


TABLE 2: Demographic characteristics, length of hospital stay and operation time of patients in laparoscopy and laparotomy groups.

\begin{tabular}{|c|c|c|c|}
\hline & Laparoscopy (n: 92) & Laparotomy (n:110) & P-value \\
\hline Patient age (years) & $33.1 \pm 4.9$ & $32 \pm 5.1$ & 0.122 \\
\hline Gravidity (n) & $3.15 \pm 1.67$ & $3.14 \pm 1.71$ & 0.947 \\
\hline Parity (n) & $1.38 \pm 1.07$ & $1.45 \pm 1.35$ & 0.671 \\
\hline Curettage & $0.24 \pm 0.6$ & $0.22 \pm 0.25$ & 0.804 \\
\hline Pregnancy week (weeks) & $6.5 \pm 1.6$ & $6.5 \pm 1.3$ & 0.818 \\
\hline Length of hospital stay (days) & $1.4 \pm 0.6$ & $3.1 \pm 0.8$ & 0.012 \\
\hline Operation time (min) & $83.3 \pm 22.3$ & $65.5 \pm 15.3$ & 0.032 \\
\hline Preoperative $\beta$-HCG level (IU/mL) & $5448 \pm 6096$ & $4032 \pm 4709$ & 0.078 \\
\hline Preoperative Hb level (gr/dl) & $11.09 \pm 1.82$ & $10.98 \pm 3.39$ & 0.790 \\
\hline Postoperative Hb level (gr/dl) & $10.3 \pm 1.05$ & $10.5 \pm 2.42$ & 0.607 \\
\hline
\end{tabular}

mission and risk factors for an EP. Admission complaints and risk factors for an EP for both groups are listed in Table 3 . When the localization of pregnancy materials in the two groups was examined, the most common localization site found in both groups was the fallopian tube. No differences at a statistical level were found between the groups for the localization site.

No complications related to the surgical intervention were observed in any of the patients. However, $9(8.1 \%)$ patients who underwent laparotomy had surgical wound infection between $6^{\text {th }}$ and $10^{\text {th }}$ postoperative days. Of the patients, three were treated with outpatient antibiotic therapy and six were treated with inpatient antibiotic therapy. All patients were successfully treated. The risk of sur- gical wound infection was higher at a statistical level in the laparotomy group than in the laparoscopy group. In the laparoscopy group, there were no complications including surgical wound infection.

In the laparotomy group, 19 (17.2\%) symptomatic patients with $\mathrm{Hb}$ level $\leq 10 \mathrm{~g} / \mathrm{dL}$ required erythrocyte suspension transfusion; 8 (9\%) patients in the laparoscopy group required erythrocyte suspension transfusion. No differences at a significant level were observed between the groups in terms of blood transfusion and intraoperative blood loss.

The localization of EP, surgical indications, blood transfusion, and postoperative complications in the two groups are shown in Table 4.

\begin{tabular}{|c|c|c|c|c|c|}
\hline \multirow[b]{3}{*}{ Admission complaints } & \multicolumn{2}{|c|}{ Laparoscopy } & \multicolumn{2}{|c|}{ Laparotomy } & \multirow[b]{2}{*}{ P-value } \\
\hline & (n: 92) & $(\%)$ & $(n: 110)$ & $(\%)$ & \\
\hline & & & & & \\
\hline Vaginal bleeding & 13 & 14.1 & 26 & 23.6 & 0.094 \\
\hline Abdominal pain & 55 & 59.8 & 58 & 52.7 & \\
\hline Pelvic pain & 21 & 22.8 & 26 & 23.6 & \\
\hline Delayed menstruation & 3 & 3.3 & 0 & 0 & \\
\hline \multicolumn{6}{|l|}{ Risk factors } \\
\hline Previous use of intrauterine device & 12 & 13 & 8 & 7.2 & 0.657 \\
\hline History of ectopic pregnancy (MTX) & 6 & 6.5 & 10 & 9 & \\
\hline History of ectopic pregnancy (operated) & 5 & 5.4 & 1 & 0.9 & \\
\hline History of pelvic inflammatory disease & 3 & 3.2 & 2 & 1.8 & \\
\hline History of abdominal surgery (including previous cesarean section) & 31 & 33.6 & 41 & 37.2 & \\
\hline History of BTL & 0 & 0 & 1 & 0.9 & \\
\hline
\end{tabular}


TABLE 4: Localizations of ectopic pregnancy, surgical indications, blood transfusion, and postoperative complications in laparoscopy and laparotomy groups

\begin{tabular}{|c|c|c|c|c|c|}
\hline & \multicolumn{2}{|c|}{ Laparoscopy } & \multicolumn{2}{|c|}{ Laparotomy } & \multirow[b]{2}{*}{ P-value } \\
\hline & (n: 92) & $(\%)$ & (n: 110) & $(\%)$ & \\
\hline \multicolumn{6}{|l|}{ Localizations of ectopic pregnancy } \\
\hline Tubal & 88 & 95.7 & 103 & 93.6 & 0.697 \\
\hline Cornual & 3 & 3.3 & 4 & 3.6 & \\
\hline Ovarian & 1 & 1.1 & 3 & 2.7 & \\
\hline \multicolumn{6}{|l|}{ Surgical indications } \\
\hline Tubal rupture (on first admission) & 36 & 39.1 & 84 & 77.3 & 0.786 \\
\hline Tubal rupture (during follow-up after MTX therapy) & 4 & 4.3 & 3 & 2.7 & \\
\hline Unruptured (MTX therapy failure) & 52 & 56 & 23 & 20 & \\
\hline Blood transfusion & 8 & 19 & 19 & 17.2 & 0.29 \\
\hline Postoperative complications & 0 & 0 & 0 & 0 & 0 \\
\hline Postoperative wound infection & 0 & 0 & 9 & 8.1 & 0.002 \\
\hline
\end{tabular}

\section{DISCUSSION}

Both laparoscopy and laparotomy are used in the surgical treatment of EP. Laparoscopic surgery is the primary modality in EP treatment in women who are hemodynamically stable. ${ }^{4}$ Laparoscopic surgery has advantages such as less blood loss, shorter surgery time, less postoperative pain, and shorter hospital stay. ${ }^{6}$ Consistent with the literature, our study found that the length of hospital stay after the operation was shorter at a significant level in the laparoscopy group than in the laparotomy group ( $\mathrm{p}=0.012$ ). However, our study found that surgery time was significantly longer in the laparoscopy group than in the laparotomy group $(\mathrm{p}=0.032)$.

It is important to decide whether laparoscopy or laparotomy is performed in hemodynamically unstable or ruptured EPs. There are publications in the literature claiming that laparoscopy is possible in women who have a ruptured EP and who are hemodynamically unstable. ${ }^{7,8}$ In our study, 36 (39.1\%) patients who had a ruptured EP at first admission and $4(4.3 \%)$ patients who had a ruptured EP during follow-up after methotrexate treatment underwent laparoscopic surgery.

While it is accepted that laparoscopic surgery is contraindicated in some cases like interstitial/ cornual pregnancy, obesity, and serious adhesions, most of these absolute contraindications are now invalid with the improvement of surgical skills and instruments in recent years. ${ }^{910}$ In our study, three (3.2\%) patients with a cornual EP underwent laparoscopic surgery and did not have any complications.

While the pneumoperitoneum established in laparoscopic surgery leads to positive intra-abdominal pressure, which reduces venous return and cardiac output, the Trendelenburg position during surgery can increase cardiac output and can provide bleeding control partially because of compression of blood vessels by an established pneumoperitoneum. ${ }^{6}$ In our study, 110 (54\%) patients underwent laparotomy while 92 (46\%) patients underwent laparoscopic surgery. In the laparoscopy group, there were no complications in patients with a ruptured EP.

Soriano et al. compared the efficacy and postoperative complications of laparoscopic surgery in 178 patients with an EP, who were hemodynamically stable or unstable. Only three patients were converted to laparotomy because of hemodynamic instability. No significant differences were detected in postoperative complications between the two groups. ${ }^{7}$ In our study, no patient in the laparoscopy group was converted to laparotomy.

Yao et al. compared laparoscopy and laparotomy in the surgical treatment of EP and concluded that both treatments had similar efficacy. ${ }^{11}$ 
Studies have shown that laparoscopic surgery provides advantages such as short hospitalization, rapid recovery, and low cost over laparotomy. ${ }^{12}$ In our study, the hospital stay was found to be shorter in accordance with that in the literature.

\section{CONCLUSION}

When surgical treatment is decided in patients with EP, laparoscopic surgery can be preferred more because it leads to less contamination, reduces hospital stay, and has a cost advantage over laparotomy. However, treatment methods can vary according to the facilities of clinics, availability of surgical instruments, and experience of physicians.

\section{Source of Finance}

During this study, no financial or spiritual support was received neither from any pharmaceutical company that has a direct connection with the research subject, nor from a company that provides or produces medical instruments and materials which may negatively affect the evaluation process of this study.

\section{Conflict of Interest}

No conflicts of interest between the authors and / or family members of the scientific and medical committee members or members of the potential conflicts of interest, counseling, expertise, working conditions, share holding and similar situations in any firm.

\section{Authorship Contributions}

Idea/Concept: Pınar Yalçın Bahat, Gökçe Turan, Berna Aslan Çetin, İbrahim Polat; Design: Pınar Yalçın Bahat, Gökçe Turan, Berna Aslan Çetin; Control/Supervision: Gökçe Turan, Berna Aslan Çetin; Data Collection and/or Processing: Pınar Yalçın Bahat, Gökçe Turan; Berna Aslan Çetin; Analysis and/or Interpretation: Berna Aslan Çetin; Literature Review: Gökçe Turan, Writing the Article: Pınar Yalçın Bahat, Gökçe Turan; Critical Review: Berna Aslan Çetin; References and Fundings: Pınar Yalçın Bahat, Gökçe Turan, Berna Aslan Çetin; Materials: Pınar Yalçın Bahat, Gökçe Turan, Berna Aslan Çetin, İbrahim Polat.

\section{REFERENCES}

1. Farquhar CM. Ectopic pregnancy. Lancet 2005;366(9485):583-91.

2. Thonneau P, Hijazi Y, Goyaux N, Calvez T, Keita N. Ectopic pregnancy in Conakry, Guinea. Bull World Health Organ 2002;80(5):365-70.

3. Condous G. Ectopic pregnancy: challenging accepted management strategies. Aust N Z J Obstet Gynaecol 2009;49(4):346-51.

4. Carson SA, Buster JE. Ectopic pregnancy. N Engl J Med 1993;329(16):1174-81.

5. Langer R, Raziel A, Ron-El R, Golan A, Bukovsky I, Caspi E. Reproductive outcome after conservative surgery for unruptured tubal pregnancy--a 15-year experience. Fertil Steril 1990;53(2):227-31.
6. Tulandi T, Kabli N. Laparoscopy in patients with bleeding ectopic pregnancy. J Obstet Gynaecol Can 2006;28(5):361-5.

7. Soriano D, Yefet $Y$, Oelsner G, Goldenberg M, Mashiach S, Seidman DS. Operative laparoscopy for management of ectopic pregnancy in patients with hypovolemic shock. J Am Assoc Gynecol Laparosc 1997;4(3):3637.

8. Sagiv R, Debby A, Sadan O, Malinger G, Glezerman M, Golan A. Laparoscopic surgery for extrauterine pregnancy in hemodynamically unstable patients. J Am Assoc Gynecol Laparosc 2001;8(4):52932.
9. Corić M, Barisić D, Strelec M. Laparoscopic approach to interstitial pregnancy. Arch Gynecol Obstet 2004;270(4):287-9.

10. MacRae R, Olowu O, Rizzuto Ml, Odejinmi F. Diagnosis and laparoscopic management of 11 consecutive cases of cornual ectopic pregnancy. Arch Gynecol Obstet 2009;280(1):59-64.

11. Yao M, Tulandi T. Current status of surgical and nonsurgical treatment of ectopic pregnancy. Fertil Steril 1997;67(3):421-33.

12. Odejinmi F, Sangrithi M, Olowu O. Operative laparoscopy as the mainstay method in management of hemodynamically unstable patients with ectopic pregnancy. J Minim Invasive Gynecol 2011;18(2):179-83. 